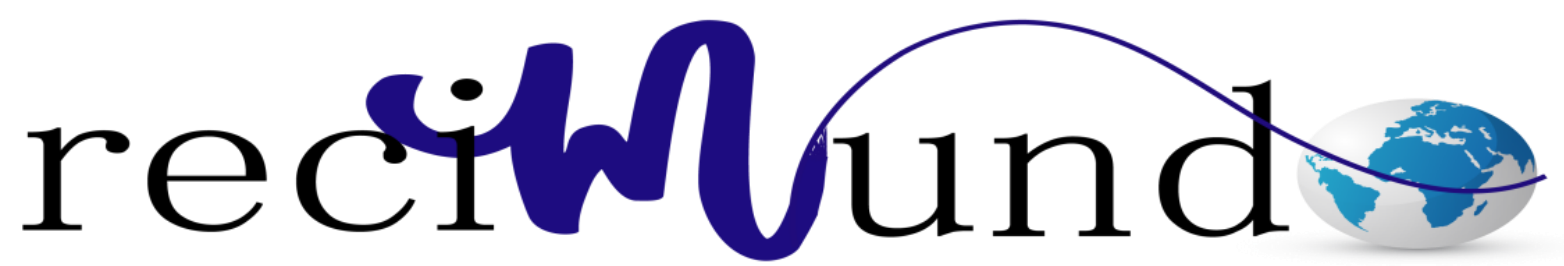

Revista Científica Mundo de la Investigación y el Conocimiento

Sonia Cedeño Loor ${ }^{\mathrm{a}}$; Eduardo Vera Muthre ${ }^{\mathrm{b}}$; Cesar Bravo Bermeo ${ }^{\mathrm{c}}$; Carmen Lazo Caicedo ${ }^{\mathrm{d}}$

Frecuencia e identificación de lesiones en accidentes de tránsito de policías motorizados. Guayaquil 2015-2016

Frequency and identification of injuries in traffic accidents of motorized police. Guayaquil 2015-2016

Revista Científica Mundo de la Investigación y el Conocimiento. Vol. 2 núm.3, julio, ISSN: 2588-073X, 2018, pp. 43-56

DOI: 10.26820/recimundo/2.(3).julio.2018.43-56

Editorial Saberes del Conocimiento

Recibido: 05/04/2018

Aceptado: 07/06/2018

Publicado: 30/07/2018

Correspondencia: leylla.cedenol@ug.edu.ec

a. Docente Carrera de Medicina. Universidad de Guayaquil; leylla.cedenol@ug.edu.ec

b. Docente Carrera de Medicina. Universidad de Guayaquil; eduardo.veram@ug.edu.ec

c. Docente Carrera de Medicina. Universidad de Guayaquil; cesar.bravob@ug.edu.ec

d. Docente Carrera de Medicina. Universidad de Guayaquil; carmen.lazoc@ug.edu.ec 


\section{Frecuencia e identificación de lesiones en accidentes de tránsito de policías motorizados. Guayaquil 2015-2016}

Vol. 2, núm. 3., (2018)

Sonia Cedeño Loor; Eduardo Vera Muthre; Cesar Bravo Bermeo; Carmen Lazo Caicedo

\section{RESUMEN}

Introducción. Los accidentes de tránsito son un problema de salud pública por su frecuencia y las elevadas cifras de morbimortalidad; de los relacionados con motos que involucra a civiles y uniformados hay escaso registro, a pesar de que empíricamente se reconoce la magnitud de su ocurrencia y el impacto sobre la salud y economía. Los agentes motorizados son un grupo vulnerable por los factores de riesgo asociados debido a las funciones que desempeñan. Objetivo. Determinar la prevalencia e identificación clínica de las lesiones de los policías motorizados que acudieron a la Emergencia del Hospital de la Policía Nacional del Ecuador, con sede en la ciudad de Guayaquil, durante el segundo semestre del año 2015 y el primer semestre del 2016. Métodos. Estudio documental de abordaje cuantitativo, descriptivo, retrospectivo, no experimental, basado en los datos de 76 historias clínicas que reposan en la sección estadística del hospital. Resultados. Alta prevalencia; varones entre 20-25años; factores de riesgo: estar en operativos, velocidad, alcohol, no usar casco; lesiones más observadas: fracturas óseas de extremidades inferiores (60) y craneales (16). Conclusión. Los policías que conducen motos están sujetos a mayores riesgos porque estando en servicio se involucran en persecuciones a velocidades superiores a las establecidas por las normas de tránsito; los policías fuera de servicio por la combinación de velocidad, ingesta de alcohol y la falta de protección de la cabeza.

Palabras claves: Accidentes de tránsito, policías motorizados. 


\title{
Frecuencia e identificación de lesiones en accidentes de tránsito de policías motorizados. Guayaquil 2015-2016
}

Vol. 2, núm. 3., (2018)

Sonia Cedeño Loor; Eduardo Vera Muthre; Cesar Bravo Bermeo; Carmen Lazo Caicedo

\begin{abstract}
Introduction. Traffic accidents are public health problems because of their frecuency and high morbidity and mortality rates; of those related to motorcycles involving civilians and uniformed personnel are scarced registered, despite the empirical recognition of the magnitude of their ocurrence and the impact on health and economy. Motorized agents are a vulnerable group because of the associated risk factors due to the functions they perform. Objective. To determine the prevalence and clinical identification the injuries of motorized pólice officers who atended the Emergency of the Hospital of the National Police of Ecuador, based in city of Guayaquil during the second half of 2015 and the first half of 2016. Methods. Documentary study of a quantitative, descriptive, retrospective, non-experimental approach based on data from the 76 clinical records that rest on the statistical section of the hospital. Results. High prevalence; males between 20-25 years; more frecuent lesions, bone fractures of the lowers limbs (60) and cranials (16); risk factors: being in operation, speed, alcohol, not wearing a helmet. Conclusion. Policemen who drive motorcycles are subject to greater riscos because being in service are involved in persecutions at speeds higher than those established by traffic regulations; the cops out of service by the combination of speed, alcohol intake and lack of protection of the head.
\end{abstract}

Keywords: Traffic accidents, motorized police. 


\section{Frecuencia e identificación de lesiones en accidentes de tránsito de policías motorizados. Guayaquil 2015-2016}

Vol. 2, núm. 3., (2018)

Sonia Cedeño Loor; Eduardo Vera Muthre; Cesar Bravo Bermeo; Carmen Lazo Caicedo

\section{Introducción.}

Hay una gran diferencia entre la moto construida por Wilhem Malbach Y Gottlieb Daimler en 1885 cuya velocidad era de $18 \mathrm{Km} /$ hora y las actuales que se fabrican en serie y desarrollan velocidades de hasta de $423 \mathrm{Km} /$ hora como la Dodge Tomahawk. Las motos son de diverso tipo y con el paso del tiempo se han convertido en un medio de transporte personal y familiar, se las utiliza para pasear, como herramienta deportiva y laboral, también para delinquir ${ }^{1}$. El mercado de motos ha crecido de forma exponencial por la necesidad de acortar distancias y tiempos en la sociedad urbanizada actual y también en la rural. Proporcionan sensación de independencia por las velocidades que desarrollan y su fácil maniobralidad. Resultan atractivas para los jóvenes y son más asequibles que los automóviles por su menor costo y llenan una necesidad de personas, grupos e instituciones.

La experiencia empírica determina que conducir motos conlleva elevado riesgo de sufrir accidentes que pueden tener consecuencias graves para la salud y la vida de conductores y acompañantes. Es un importante factor de muerte prematura y discapacidad a nivel local, regional y global ${ }^{2-3}$. Se ha demostrado que en los accidentes de motos están casi siempre presente tanto el comportamiento enervado de los conductores como las velocidades extremas que imprimen a los aparatos, factores concurrentes observados de manera similar en diferentes tipos de accidentes de tráfico vial ${ }^{4}$. Son los jóvenes los más frecuentemente accidentados por la intrepidez propia de la etapa de vida en que no se es consciente de los peligros inherentes de una maquina frágil e insegura y de las consecuencias relacionadas con las colisiones de motos en las que son parte activa. 


\section{Frecuencia e identificación de lesiones en accidentes de tránsito de policías motorizados. Guayaquil 2015-2016}

Vol. 2, núm. 3., (2018)

Sonia Cedeño Loor; Eduardo Vera Muthre; Cesar Bravo Bermeo; Carmen Lazo Caicedo

La literatura mundial y las estadísticas oficiales de la Organización Mundial de la Salud ${ }^{3}$ muestran datos alarmantes de las cifras de morbimortalidad resultado de accidentes en carreteras y calles de pueblos y ciudades y señala que las colisiones, muertes, heridos y discapacitados resultantes, son más frecuentes en los países de regiones con menor nivel de desarrollo que en los desarrollados. Resalta también que un porcentaje importante de estas cifras corresponden a las determinadas por accidentes de motos.

El Ecuador no es ajeno a esta situación calificada como endémica y son cada vez más graves y más frecuentes los accidentes de tránsito que cobran vidas, discapacitan y enlutan familias. Si bien en las zonas urbanas ocurren accidentes graves, es en las carreteras que interconectan poblaciones donde se dan con más frecuencia y los que abonan las cifras mayores son los buses interprovinciales que colisionan frontalmente o se desbarrancan como ocurre en las zonas altas de la región interandina en las que intervienen factores relacionados con el vehículo, ejemplo, llantas lisas, frenos en mal estado, dirección y cambios en mal estado; con el conductor, el cansancio por horas extras de conducción en carreteras con poca visibilidad y también por velocidades no permitidas.

Dentro de este marco de accidentes de tráfico vial, los accidentes en moto por no generar el impacto visual y real en la sociedad- en las motos se mueren, salen heridos o discapacitan 1 o 2 personas-, como si lo hacen los accidentes de transporte de conglomerados humanos, los investigadores analizan y reflexionan con más frecuencia sobre accidentes graves y se enfocan menos en los que están involucradas las motos en las que intervienen factores de desencadenamiento, algunos similares y otros diferentes 


\section{Frecuencia e identificación de lesiones en accidentes de tránsito de policías motorizados. Guayaquil 2015-2016}

Vol. 2, núm. 3., (2018)

Sonia Cedeño Loor; Eduardo Vera Muthre; Cesar Bravo Bermeo; Carmen Lazo Caicedo

Hay estudios de accidentes en motos de la sociedad civil y está demostrado que el estado de ánimo, la velocidad y la indiferencia a las medidas de seguridad intervienen en la generación de los mismos 5 . Pero más escasa es la información sobre los accidentes en moto de los miembros de las instituciones responsables de la seguridad de bienes y vida de la ciudadanía que, mantienen grupos dedicados exclusivamente al patrullaje y operativos en motos, reconocidos como motorizados.

La observación que en la sala de emergencia del hospital de la Policía Nacional de Guayaquil recibe y atiende numerosos motorizados accidentados tanto en servicio como los que no están, planteo la interrogante de establecer con cifras la frecuencia y la identificación de las heridas más frecuentes de los accidentados por lo que se planifico una investigación cuyo objetivo era demostrar con números la observación empírica de la elevada frecuencia de accidentes en moto de los policías e identificar el tipo de lesiones que experimentan con más frecuencia, cuyos resultados fundamentarían el propósito de insistir sobre las medidas de prevención de accidentes que la institución tiene normadas y que son inobservadas como lo demuestran la observación y las historias clínicas.

\section{Métodos.}

Tipo de investigación descriptiva, retrospectiva, transversal, documental, que tiene como fuente de información secundaria de 76 historias clínicas de policías motorizados tanto en servicio como fuera de servicio, que experimentaron accidentes y fueron atendidos en la emergencia del Hospital de la Policía Nacional ubicado en la ciudad de Guayaquil, durante el último semestre del 2015 y el primer semestre del 2016. Unidades de análisis policías 


\section{Frecuencia e identificación de lesiones en accidentes de tránsito de policías motorizados. Guayaquil 2015-2016}

Vol. 2, núm. 3., (2018)

Sonia Cedeño Loor; Eduardo Vera Muthre; Cesar Bravo Bermeo; Carmen Lazo Caicedo

motorizados. Universo-muestra lo constituyeron todos los motorizados accidentados que concurrieron a la emergencia de la unidad hospitalaria de la Policía Nacional.

Se registró la edad, el informe de si los policías estaban en patrullaje o no, el tipo de lesiones al egreso, si el conductor había consumido alcohol, si usaba casco al momento del accidente y la velocidad inferida por la labor que realizaban al momento o por la información que proporcionaban los accidentados.

La revisión, análisis, agrupamiento y categorización de los datos fue guiado por el tipo de lesiones observadas y el diagnóstico elaborado al alta u hospitalización de los pacientes así como por los días de hospitalización. En cuanto los factores de riesgo se consideraron la edad, la alta velocidad, participar en operativos, portar casco e ingesta de alcohol. 
Frecuencia e identificación de lesiones en accidentes de tránsito de policías motorizados. Guayaquil 2015-2016

Vol. 2, núm. 3., (2018)

Sonia Cedeño Loor; Eduardo Vera Muthre; Cesar Bravo Bermeo; Carmen Lazo Caicedo

\section{Resultados.}

FIGURA.1

\section{DIAGNÓSTICO DE EGRESOS 2015}

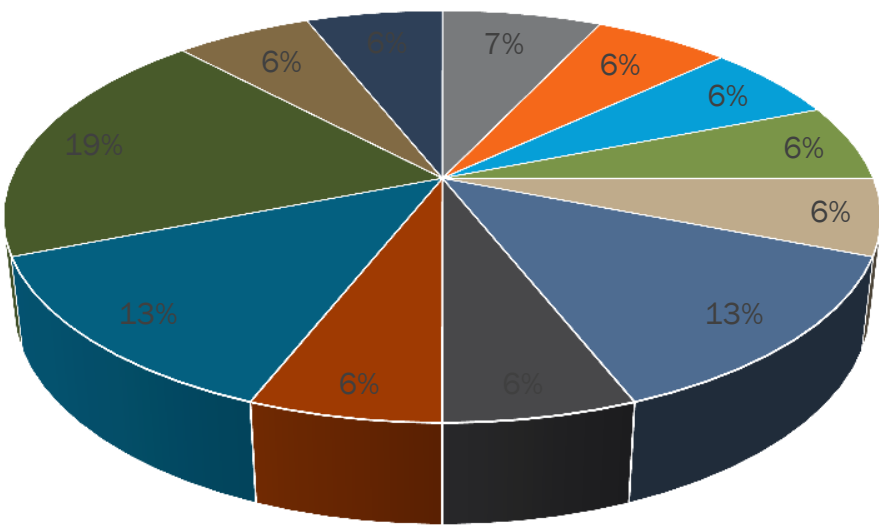

- FRACTURA DE LAS OTRAS PARTES DE LA MUÑECA Y MANO

- FRACTURA DE LA DIAFISIS DEL FEMUR

- SECUELAS DE TRAUMATISMO QUE AFECTAN MULTIPLES REGIONES DEL CUERPO

- TRAUMA SUPERFICIAL DEL HOMBRO Y DEL BRAZO

- OTROS TRAUMATISMO DE LA CABEZA

- HEMORRAGIA SUBARACNOIDEA

- FRACTURA DEL SUELO DE LA ORBITA

- FRACTURA DE OTRO DEDO DE LA MANO

- FRACTURA DEL CRANEO Y DE LOS HUESOS DE LA CARA

- FRACTURA DEL SUELO DE LA ORBITA

- FRACTURA DE OTRO DEDO DE LA MANO

- FRACTURA DE LA DIAFISIS DE LA TIBIA 
Frecuencia e identificación de lesiones en accidentes de tránsito de policías motorizados. Guayaquil 2015-2016

Vol. 2, núm. 3., (2018)

Sonia Cedeño Loor; Eduardo Vera Muthre; Cesar Bravo Bermeo; Carmen Lazo Caicedo

\section{FIGURA 2}

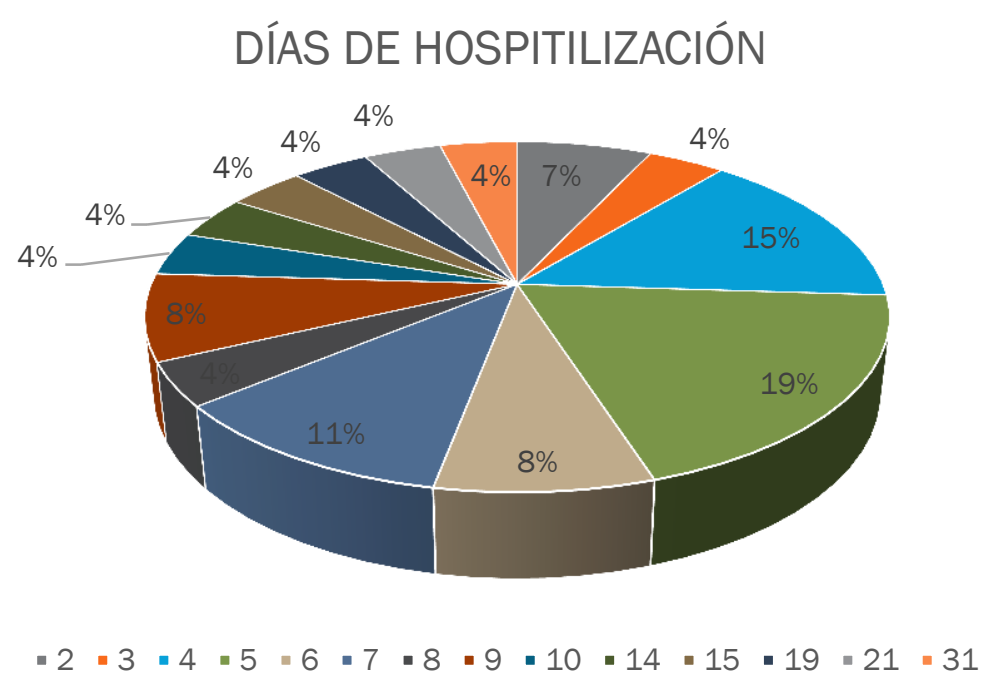

FIGURA 3

\section{DIAGNÓSTICO DE EGRESOS 2016}

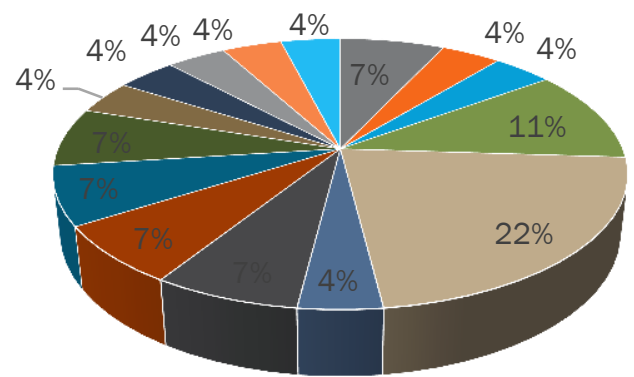

- TRAUMA DEL PIE Y DEL TOBILLO

- FRACTURA DEL SUELO DE LA ORBITA

- AMPUTACION TRAUMATICA DE UNO O MAS DEDOS DEL PIE

- FRACTURA MULTIPLE DEL FEMUR

- TRAUMATISMO MULTIPLE

- FRACTURA ARTICULAR EN MESETA TIBIAL

- FRACTURA DEL MALEOLO EXTERNO

- TRAUMA MULTIPLE EN LA RODILLA

- FRACTURA DE LA TIBIA

- FRACTURA DE HUMERO

- FRACTURA DEL PERONE SOLAMENTE

- FLEBITIS Y TROMBOFLEBITIS DE VASOS SUPERFICIALES DE LOS MIEMBROS INFERIORES

- TRUMATIMO DEL RIÑON

- CONTUSION DE LA PIERNA

- FRACTURA DE LA CLAVICULA 
Frecuencia e identificación de lesiones en accidentes de tránsito de policías motorizados. Guayaquil 2015-2016

Vol. 2, núm. 3., (2018)

Sonia Cedeño Loor; Eduardo Vera Muthre; Cesar Bravo Bermeo; Carmen Lazo Caicedo

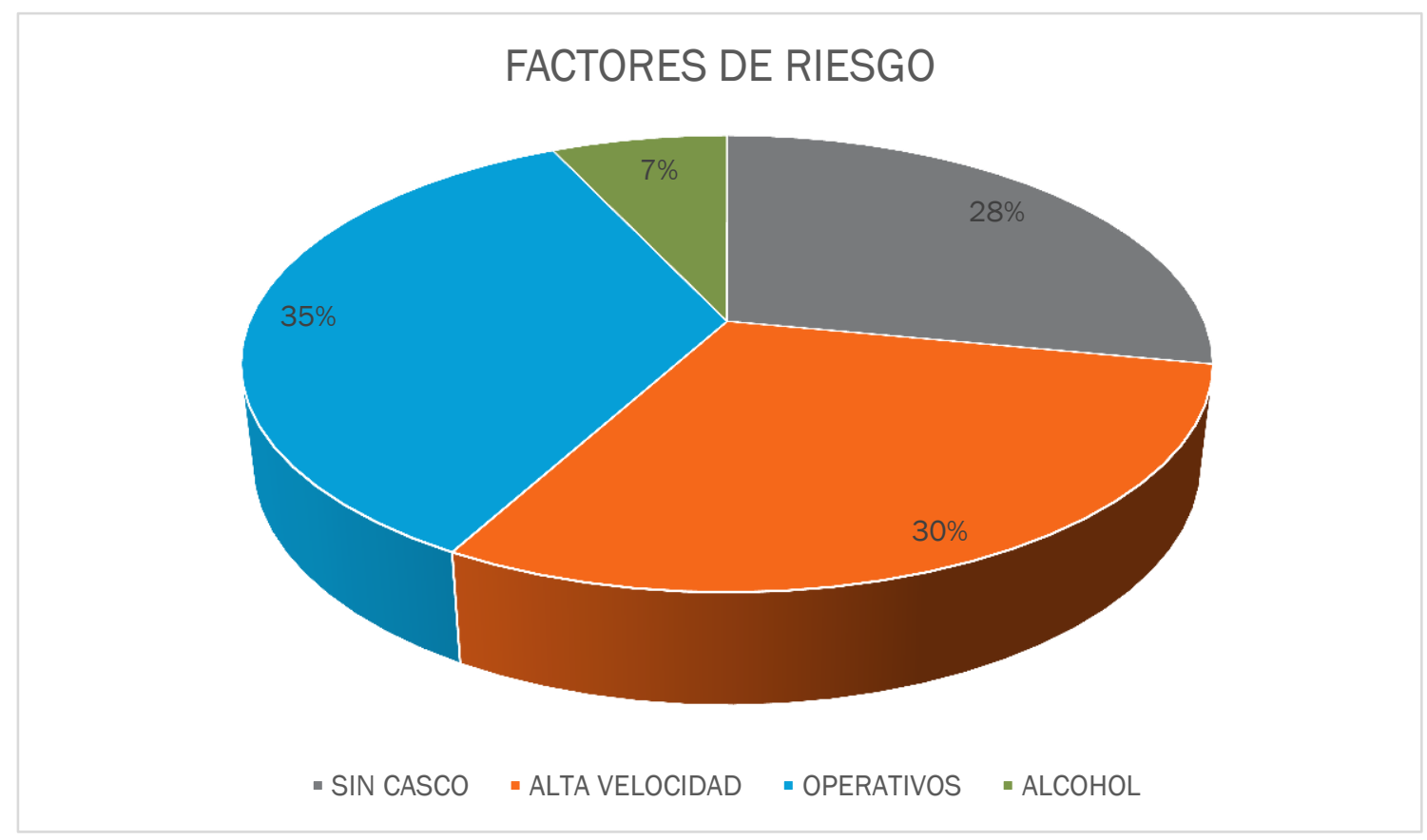

FIGURA 4

FIGURA 5

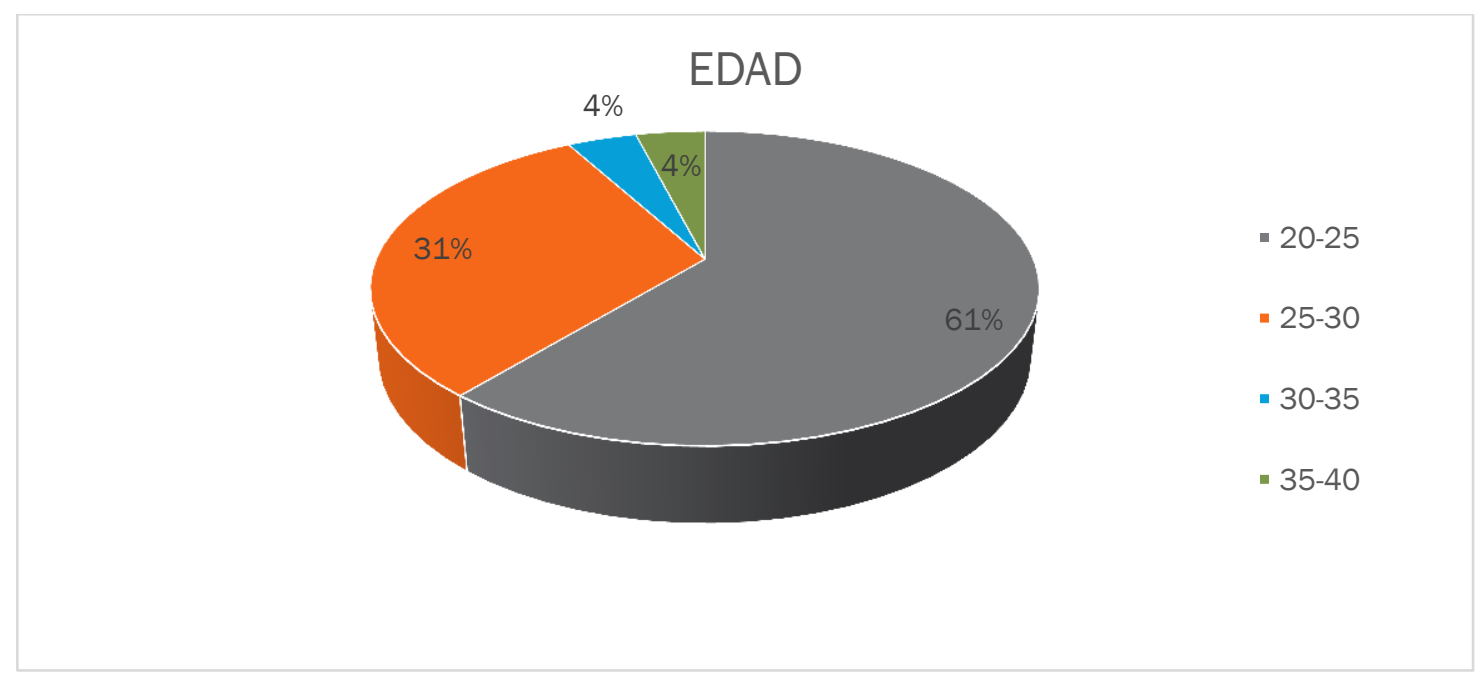




\section{Frecuencia e identificación de lesiones en accidentes de tránsito de policías motorizados. Guayaquil 2015-2016}

Vol. 2, núm. 3., (2018)

Sonia Cedeño Loor; Eduardo Vera Muthre; Cesar Bravo Bermeo; Carmen Lazo Caicedo

\section{Conclusión.}

Los traumas de las extremidades inferiores y de cráneos son los más frecuentes, reporte coincidente con el estudio descriptivo de Aye Moe Moe Lain ${ }^{6}$, y un número menor de lesiones de diferente expresión clínica que involucraron partes blandas, musculo esqueléticas y órganos internos, fueron observados. Los traumatismos múltiples involucrando varios huesos e incluso órganos internos, se relacionan con la magnitud del impacto y la velocidad de los vehículos en contacto

Los huesos más afectados fueron los huesos del pie, frecuencia similar a la reportada en el estudio de Jeffers, R.F, y colaboradores ${ }^{7}$ que, reportan como lesión más común la fractura metatarsiana y aunque estas injurias están asociadas con baja mortalidad, requieren pronta evaluación y tratamiento para evitar morbilidad a largo plazo y discapacidad, menos frecuente las lesiones de peroné, rodilla, fémur y la tibia, este último dato contrasta con la alta frecuencia de lesión ósea tibial registrada por Almeida, $\mathrm{M}^{8}$, en su revisión de 81 pacientes con $47 \%$ de fracturas expuestas de tibia. Lesiones de la cabeza y suelo de la órbita se describen con frecuencia y se relacionaron con la falta de protección por el casco y son las que mayores consecuencias acarrean en el mediano y largo plazo así como las que mayores costos demandan como asevera el informe del U.S. Department of Transportación. $2015^{9-10}$.

No se espera que el tipo de lesiones de los accidentes de motos que involucran a los policías sean diferentes a las de los civiles, porque las variables que los determinan son las mismas y los actores difieren básicamente en el uniforme. Con relación a la frecuencia se supone los policías debieran tener menos accidentes por su formación profesional con más conocimiento 


\section{Frecuencia e identificación de lesiones en accidentes de tránsito de policías motorizados. Guayaquil 2015-2016}

Vol. 2, núm. 3., (2018)

Sonia Cedeño Loor; Eduardo Vera Muthre; Cesar Bravo Bermeo; Carmen Lazo Caicedo

y respeto de las normas y principios, respondiendo a su condición de elementos clave de la

sociedad. Pero, hay que considerar que el grupo motorizado creado para dar auxilio y protección más inmediata tiene mayor riesgo de accidentes por los operativos que realizan, en la que la alta velocidad es necesaria para alcanzar los objetivos de este tipo de misiones, como se observó en esta revisión en la que concurrieron los dos factores con porcentajes similares de ocurrencia. La integridad física del motorizado depende, fundamentalmente, de la precaución de conducir una maquina adecuada a la experiencia del conductor, respetar las señales de tránsito y no sobrepasar las velocidades permitidas, tener siempre puesto el casco protector y no conducir bajo los efectos del alcohol ${ }^{11}$ Las estadística de muerte de la OMS-300.000 por año- debería ser un advertencia a considerar.

Esta investigación responde a la observación de la elevada frecuencia de accidentes de los policías motorizados atendidos en la emergencia del Hospital de la Policía Nacional de la ciudad de Guayaquil, durante el segundo semestre del 2015 y el primero del 2016. Es una revisión cuantitativa de tipo exploratorio dado que hay muy poca o ninguna información sobre el tema específico_accidentes de policías motorizados_en contraste con la amplia y actualizada sobre los accidentes en moto de la población civil, por lo que no es posible establecer analogías entre resultados y conclusiones que afectan por un lado a civiles y por otro a uniformados. Sin embargo, la investigación pone de relieve que los accidentes de motorizados en la ciudad de Guayaquil es una realidad con honda repercusión personal, familiar e institucional. Significa para los involucrados, aun en los casos de lesiones leves o moderadas ausencias en sus trabajos y registros negativos para sus hojas de vida cuando los accidentes ocurren fuera de servicio y se dan por negligencia en unos casos, como no usar casco protector o irresponsabilidad, cuando los 


\section{Frecuencia e identificación de lesiones en accidentes de tránsito de policías motorizados. Guayaquil 2015-2016}

Vol. 2, núm. 3., (2018)

Sonia Cedeño Loor; Eduardo Vera Muthre; Cesar Bravo Bermeo; Carmen Lazo Caicedo

accidentes ocurren bajo el efecto del alcohol; en los casos con lesiones graves y secuelas permanentes los policías se ven obligados a retirarse o son dados de baja, situación que afecta a la familia por la vulnerabilidad adquirida debido a la perdida de trabajo de los, en muchos casos, jefes de familia; igual la institución es afectada por los costos de atención médica y por la pérdida de un recurso humano en el que se ha invertido tiempo y dinero para su formación. Constituye, además, una alerta para que las autoridades policiales intervengan con reuniones informativas, de concienciación y prevención, así como el cumplimiento riguroso de sanciones para aquellos policías a quienes se demuestre negligencia o irresponsabilidad. Finalmente, esta revisión documental constituye punto de partida para estudios prospectivos que aborden el tema con enfoque objetivo y detallado de las variables que se conceptúan en los accidentes de motos: velocidad, uso de casco, ingesta de alcohol, estar de guardia en operativos o no, otras 


\section{Frecuencia e identificación de lesiones en accidentes de tránsito de policías motorizados. Guayaquil 2015-2016}

Vol. 2, núm. 3., (2018)

Sonia Cedeño Loor; Eduardo Vera Muthre; Cesar Bravo Bermeo; Carmen Lazo Caicedo

\section{Bibliografía.}

1. Wigan M. Motorcycle as Transport. Managing Mobility. 2001. Disponible en: http://www.geocities.com/mwigan/geoMRW.html

2. World Health Organization. Global status report on road safety 2015. Italia: World Health Organization; 2015.

3. World Health Organization. Health topics: Road traffic injuries. Italia: World Health Organization, 2016.

4. Chu Cong M, Sano K, Matsumoto S. The speed, flow and headway analyses of motorcycle traffic. Jour of the East Asia Soc for Trans Studies. 2005; 6:1496-1508.

5. Lili X, Yao Z, Liping L. Risk Factors for Motorcycle related Severe Injuries in a Mediun-size City in China. AIMS Public Health, 2016; 3(4): 907-922.

6. Aye Moe Moe Lain, Yi Yi Win, Thinzar Aung, Thit Lwin. 514 Factors influencing motorcycle accidents in nay Pyi Taw, Myanmar. Traffic Safety. Post Mon 1.12; 22(2).

7. Jeffers RF, Tan HB, Nicolopoulos C, Kamath R, Giannoudis PV. Prevalence and patterns of foot injuries following motorcycle trauma. J Orthop Trauma. 2004; 18(2):87-91.

8. Almeida M, Do Nacimiento J, Vieira B. Clinical and demographic study on open fractures caused by motorcycle traffic. Acta Ortop Bras. 2014; 22(4): 214-8. Disponible en: https://www.ncbi.nlm.nih.gov/pmc/articles/PMC4167047/

9. US. Deparment of Transportation. National Highway Traffic Safety Administration,Estimatying Lives and Cost Saved by Helmets Motorcycle with Update Economic Cost Information. 2015

10. Liu BC, Ivers R, Norton R, Boufous S, Lo SK. Helmets for preventing injury in motorcycle riders. Cochrane Database Syst Rev. 2008; 23(1):

11. Lin MR, Kraus JF. A review of risk factors and patterns of motorcycle injuries. Accid, Anal Prev. 2009; 41(4): 710-22 